\title{
Sudden onset of a huge subcapsular renal hematoma following minimally invasive ureteroscopic holmium laser lithotripsy: A case report
}

\author{
PENG ZHANG and WAN-LI HU \\ Department of Urology, Zhongnan Hospital, Wuhan University, Wuhan, Hubei 430071, P.R. China
}

Received July 29, 2014; Accepted April 2, 2015

DOI: $10.3892 /$ etm.2015.2460

\begin{abstract}
The postoperative complication rate of ureteroscopic lithotripsy (URSL) is low, and the most frequent complication is fever. Thus, urological surgeons often neglect or are unaware of subcapsular renal hematoma (SRH) as a rare complication following URSL. Although a SRH after undergoing URSL is uncommon, the occurrence may be fatal. The current study reports on a rare life-threatening case that occurred recently at Zhongnan Hospital (Wuhan, China). A 24-year-old male patient presented with a large SRH $(11 \times 14 \times 11 \mathrm{~cm})$ after undergoing a failed, but minimally invasive URSL with a holmium laser. The presence of the large SRH was confirmed by computerized tomography imaging. The patient underwent conservative management, which prevented hematoma enlargement, and no further treatment was conducted. In conclusion, SRH, although rare following URSL, should be noted by the urological surgeon.
\end{abstract}

\section{Introduction}

Shock wave lithotripsy (SWL), trauma and renal angiographic procedures are all known to cause renal hematoma (1). However, a renal hematoma may also occur spontaneously in patients with a malignancy or in patients receiving anticoagulation therapy. Although the incidence is rare, the occurrence of a renal hematoma can be fatal (2). Ureteroscopic lithotripsy (URSL) as a transurethral, minimally invasive and efficient procedure has become a regular operation for treatment of ureteral calculi. For example, the ureteroscopic holmium laser lithotripsy is the current technique of choice for the treatment of ureteric stones (3). The postoperative complication rate of URSL is low, with the most frequent complication being a fever $(4,5)$. Therefore, it

Correspondence to: Mr. Wan-Li Hu, Department of Urology, Zhongnan Hospital, Wuhan University, 169 Donghu Road, Wuhan, Hubei 430071, P.R. China

E-mail: 92011552730@sina.com

Key words: ureteral calculus, ureterorenoscopy, subcapsular renal hematoma, conservative management is easy for urological surgeons to neglect or be unaware of a subcapsular renal hematoma $(\mathrm{SRH})$ as a rare complication following URSL therapy.

With a kidney fracture or spontaneous renal hemorrhage, SRH will occur. It can cause hemorrhagic shock when the active bleeding fails to be prevented. Even when the active bleeding is controlled, the SRH will lead to high blood pressure and subsequent renal failure since the active subcapsular bleeding will accumulate around the kidney and compressed it for a substantial amount of time. Engel and Page (6) first reported a clinical case of hypertension caused by subcapsular renal hematoma and it improved following nephrectomy. The present study reports such a case, in which a young male patient suffered from a SRH two days after undergoing failed URSL therapy with a holmium laser (UHL).

\section{Case report}

Written informed consent was obtained from the patient, a 24-year-old male patient, complaining of distending pain in the left lumber region for 10 days and diagnosed with moderate dilatation of the upper ureter, left hydronephrosis and left kidney stones by ultrasonography, who was admitted to Zhongnan Hospital (Wuhan, China). A contrast-enhanced computerized tomography (CT) scan confirmed two calculi in the left proximal ureter, which measured $1 \times 0.8 \times 0.5 \mathrm{~cm}$ and $0.5 \times 0.5 \times 0.8 \mathrm{~cm}$, and moderate left hydronephrosis (Fig. 1). In addition, the contrast-enhanced $\mathrm{CT}$ scan revealed good renal parenchyma and function. The patient had undergone a successful UHL due to the identification of a left ureteral calculus three years previously; however, the patient had no other disease.

Holmium laser lithotripsy (PowerSuite $60 \mathrm{~W}$; Lumenis, Inc., San Jose, CA, USA) under a rigid ureteroscope was recommended for treatment. Following the administration of intravenous anesthesia (remifentanil, $2 \mu \mathrm{g} / \mathrm{kg}$; Rui Jie Ren Fu Inc., Yi Chang, China and propofol, $1.0 \mathrm{mg} / \mathrm{kg}$; De Pu Li Ma; AstraZeneca, Shanghai, China), the patient was placed in the lithotomy position. A super lubricious guide wire (ureteral safety wire guide introducer set; Cook Urological Inc; Spencer, IN, USA) was inserted into the left ureter though the ureteral orifice, after which a $6.5-\mathrm{F}$ rigid tapered ureteroscope (Richard Wolf Medical Instruments Corporation, Vernon Hills, IL, USA) was advanced forward 


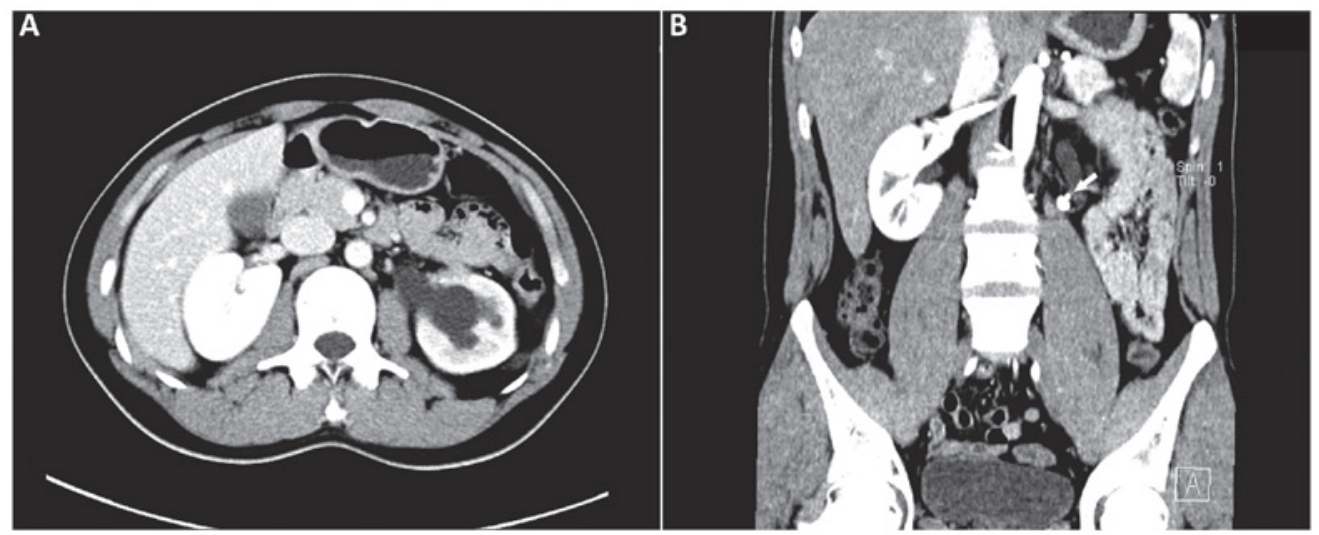

Figure 1. Images captured at the original presentation. Contrast-enhanced computerized tomography scans show (A) moderate left hydronephrosis and (B) two calculi in the left proximal ureter (arrow).
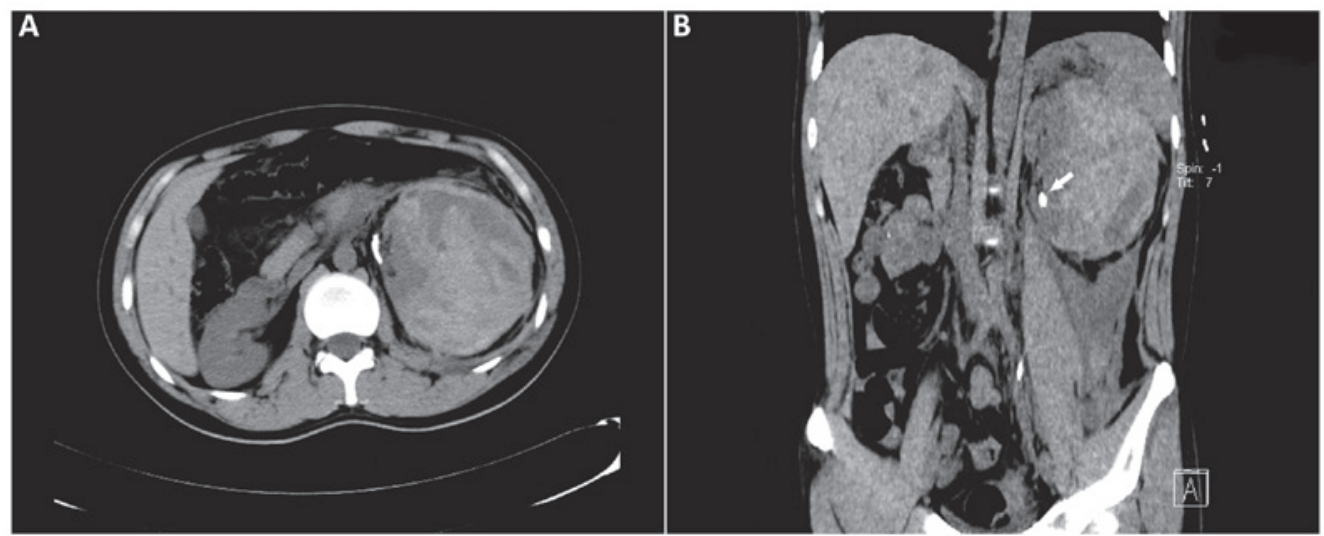

Figure 2. Images captured $36 \mathrm{~h}$ after the original presentation. Computerized tomography scans demonstrate (A and B) a huge subcapsular hematoma of the left kidney and (B) the stone in the renal pelvis from the left proximal ureter (arrow).

slowly and carefully until the stones were located. However, the treatment failed to fragment the stones completely even when the repetition rate of the laser energy was increased from 35 to $40 \mathrm{~Hz}$. In addition, the bigger stone moved to the pelvis, while the smaller calculus was crushed. To force the stone to move out from the renal pelvis, intravenous administration of $20 \mathrm{mg}$ furosemide (Luo Fu Shang Zhu She Ye, Luo Fu Shang Guo Yao Inc., Guangzhou, China) was applied, and a lower hip position and a high flushing pressure of the irrigation system were responded empirically. However, the stone was ultimately lost in the endoscopic view. A 5 FR-26-cm Double J stent (ureteral stents 7F; Bard International Inc., Karlsruhe, Germany) was placed routinely and the procedure was terminated.

At 22:30 on the following day, the patient complained of pain in the left lumber region; thus, diclofenac sodium suppositories were applied as a symptomatic treatment. After $2 \mathrm{~h}$, the pain returned and a somatoscopy indicated abdominal tenderness and rebound pain. Considering the diagnosis of an acute abdomen initially, an emergency examination was conducted. The routine blood tests revealed a blood leukocyte count of $13.30 \times 10^{9} / 1$ and a hemoglobin level of $90 \mathrm{~g} / 1$. The emergent abdominal CT scan revealed a huge subcapsular hematoma $(11 \times 14 \times 11 \mathrm{~cm})$ of the left kidney and the stone in the renal pelvis from the left proximal ureter (Fig. 2). Vital signs, urine volume and routine blood tests were monitored, and $500 \mathrm{ml}$ hydroxyethyl starch (Nanjing Zhengda Tianqing Pharmaceutical Co. Ltd., Nanjing, China) (intravenously guttae), 2 mg tramadol (Shu Min Grunenthal GmbH, Aachen, Germany) (intramuscular), $4 \mathrm{~g}$ piperacillin-tazobactam (Tazocin; Wyeth Lederle SPA Inc., Catania, Italy), $250 \mathrm{ml}$ normal saline (Sheng Li Iv Hua Na Rong Ye, Cheng Xin Yao Ye Inc., Shang Dong, China) (intravenously guttae) and 4 units Hemocoagulase Agkistrodon (Shu Ning, Kang Cheng Yao Ye Inc., Beijing, China) (intravenously guttae) were applied immediately. The patient was shown to respond to the conservative management, and the hemoglobin level remained at $82 \mathrm{~g} / 1$ over the subsequent $12 \mathrm{~h}$. The patient presented with a low-grade fever $\left(37.5^{\circ} \mathrm{C}\right)$ over several days as a result of hematoma absorption; however, further hospitalization was uneventful. After 3 weeks, a CT scan clearly showed that the left kidney was compressed to one side by the large surrounding unabsorbed hematoma. The patient attended a six-month follow-up once out of hospital and no particular event was found. In this case, however, the large SRH was mostly absorbed after six months and no evident hypertension or renal failure occurred. The invasive operation for the remaining hematoma was not considered, although this may have been due to the young and healthy condition of the patient. 


\section{Discussion}

When a SRH occurs, the renal parenchyma is usually compressed by the hemorrhage filled in the subcapsular area of the kidney, which is a potential space where fluid can accumulate (2). Furthermore, no coagulation disorders or other diseases that cause bleeding were identified in the present case and the possibility of spontaneous SRH was low $(1,2)$. Recently, URSL has become a common technique for the treatment of ureteric stones due to the low complication and high relative efficiency rates $(4,5)$. However, the presence of a SRH following URSL therapy has been rarely reported in the literature; thus, understanding this potential fatal risk is of great clinical significance (7).

In the present study, the clinical presentations and conservative treatment of a SRH following a failed UHL procedure in a young male patient were discussed. The sudden onset of severe pain in the flank was associated with the appearance of a SRH and the presentation of acute abdomen; abdominal tenderness and rebound pain following surgery were major clinical presentations in the patient. The hemoglobin level decreased markedly and the patient presented signs of blood loss. In general, contrast-enhanced CT scans were required for a confirmed diagnosis, in which the extent of the renal injuries and the diameter of the hematoma were assessed accurately, and the presence of active bleeding was determined. On an enhanced CT scan, an irregular, heterogeneous area around the kidney, with an attenuation value less than that of the artery or renal parenchyma, is known to indicate a SRH without active bleeding (8). In the present case, CT imaging was undertaken to clarify the diagnosis of an acute abdomen as quickly as possible, since a similar case had not been experienced previously, and the bleeding was controlled under a conservative management regimen.

In the patient discussed in the present study, there was no evident trauma caused by the UHL, even though the ureteric stone was unable to be fragmented and ultimately moved to the renal pelvis. However, it was hypothesized that the wire used to guide the rigid ureteroscope increased the intrarenal pressure, which led to forniceal rupture and separation of the capsule from the parenchyma, and subsequently the development of a hematoma. No coagulation disorders or other diseases that cause bleeding were identified in the present case, spontaneously SRH may be not.

Treatment protocols for SRH should be discussed. When patients are without signs for active bleeding, a conservative manner should be used to treat the SRH $(1,9)$. With the exception of contrast-enhanced CT, CT scan, ultrasound, routine blood tests and coagulation status, creatinine levels, blood urea nitrogen levels, electrolyte balance and urine tests should be included in diagnostics (1). Krishnamurthi and Streem found that the mean time of hematoma resolution was 13.2 months following conservative treatment, without adverse effects on blood pressure or renal function (9). However, the SRH cases included in this prospective study were small $(<5 \mathrm{~cm})$. Engel and Page were the first to describe a clinical case of hyperten- sion caused by SRH, which improved following a nephrectomy. A subcapsular hematoma usually compressed the kidney and was associated with high blood pressure and occasional renal failure in the patient (6). Percutaneous drainage or the use of percutaneous pigtail catheters, laparoscopic decortication, or the combined use of percutaneous drainage and urokinase injection may promote hematoma resolution in patients with stable vital signs; however, this therapy may induce unbearable pain or renal compression even with a solitary kidney (10). When active bleeding fails to be controlled by conservative management, or when there are unstable signs of blood loss, a quick and efficient treatment method should be conducted, such as a superselective embolization under renal artery digital subtraction angiography or open surgery (11). In the present case, due to the stable hematoma without evidence of active bleeding and the positive response to conservative management, invasive surgery to drain and eliminate the hematoma was not undertaken. The patient was required to undergo a 6-month follow-up.

In conclusion, not only SWL, trauma and renal angiographic procedures are causative factors for a renal hematoma. SRH should be considered as a complication following URSL, even though occurrence is not common.

\section{References}

1. Schnabel MJ, Gierth M, Chaussy CG, Dötzer K, Burger M and Fritsche HM: Incidence and risk factors of renal hematoma: A prospective study of $1,300 \mathrm{SWL}$ treatments. Urolithiasis 42: 247-253, 2014.

2. Chao YC, Ming HL, Yeu CC and Sun YC: Spontaneous bilateral renal subcapsular hematoma as a possible complication of myeloproliferative disorders. J Med Sci 29: 273-275, 2009.

3. Seitz C, Tanovic E, Kikic Z and Fajkovic H: Impact of stone size, location, composition, impaction, and hydronephrosis on the efficacy of holmium:YAG-laser ureterolithotripsy. Eur Urol 52: 1751-1757, 2007.

4. de la Rosette J, Denstedt J, Geavlete P, et al: The clinical research office of the endourological society ureteroscopy global study: Indications, complications and outcomes in 11,885 patients. J Endourol 28: 131-139, 2014.

5. Chiu PK, Chan CK, Ma WK, To KC, Cheung FK and Yiu MK: Subcapsular hematoma after ureteroscopy and laser lithotripsy. J Endourol 27: 1115-1119, 2013.

6. Engel WJ and Page IH: Hypertension due to renal compression resulting from subcapsular hematoma. J Urol 73: 735-739, 1955.

7. Meng H, Chen S, Chen G, Tan F, Wang C and Shen B: Renal subcapsular hemorrhage complicating ureterolithotripsy: An unknown complication of a known day-to-day procedure. Urol Int 91: 335-339, 2013.

8. Belville JS, Morgentaler A, Loughlin KR and Tumeh SS: Spontaneous perinephric and subcapsular renal hemorrhage: Evaluation with CT, US and angiography. Radiology 172: 733-738, 1989.

9. Krishnamurthi V and Streem SB: Long-term radiographic and functional outcome of extracorporeal shock wave lithotripsy induced perirenal hematomas. J Urol 154: 1673-1675, 1995.

10. Xu L and Li G: Life-threatening subcapsular renal hematoma after flexible ureteroscopic laser lithotripsy: Treatment with superselective renal arterial embolization. Urolithiasis 41: 449-451, 2013.

11. Shen Z, He W, Liu D, Pan F, Li W, Han X and Li B: Novel technique for the treatment of large subcapsular renal hematoma: Combined use of percutaneous drainage and urokinase injection. Int Urol Nephrol 46: 1751-1755, 2014. 